\title{
Comprender para aprender: Un programa para mejorar la comprensión y el aprendizaje de textos informativos
}

\author{
Eduardo Vidal-Abarca \\ Ramiro Gilabert
}
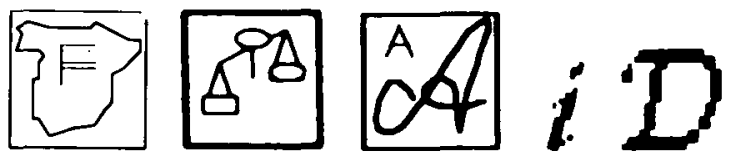

Aunque los objetivos curriculares actuales se plantean la enseñanza del lenguaje escrito de modo que permita utilizarlo como instrumento para el aprendizaje, el becho es que esta enseñanza de la lectoescritura como medio se contempla como un fin en si mismo, es decir, con materiales y contenidos literarios y no de areas disciplinares específicas en que el niño se tendrá que mover a lo largo de toda la escolaridad. En este artículo se presenta un programa experimentado con éxito en las áreas de ciencias naturales y sociales para mejorar en los alumnos estas necesarias estrategias de comprensión y estudio.

\section{INTRODUCCION}

Una de las quejas más frecuentes entre los profesores de todos los niveles educativos es que sus alumnos no comprenden lo que leen o no saben aprender autónomamente a partir de los libros de texto. Estas quejas suelen comenzar prácticamente desde el mismo momento en que se exige a los chicos que estudien un texto y sean capaces de recordar lo que dice, de responder a unas preguntas o de efectuar un resumen del contenido, lo cual suele coincidir con los últimos cursos de lo que actualmente es el Ciclo Medio de la EGB, incrementándose los problemas conforme se avanza en la escolaridad. Cuando se comienza a analizar las causas de esta situación surge una explicación plausible si se contempla la enseñanza de la comprensión lectora en el currículum escolar. Tanto en los nuevos diseños curriculares (MEC, 1989), como en los antiguos, la comprensión lectora se enmarca dentro del área de lengua y literatura. Así, entre los objetivos gene- 
rales del área que señalan los nuevos diseños curriculares se encuentran: comprender mensajes orales y escritos, utilizar la lectura con finalidades diversas, y utilizar el lenguaje escrito como instrumento de aprendizaje. Cuando revisamos el contenido de los distintos bloques temáticos apreciamos que se alude a aspectos conceptuales tales como «textos literarios y no literarios», «tipos y formas de discurso en la comunicación escrita», y sobre todo se mencionan destrezas tales como «análisis de los aspectos formales de los diferentes textos", "utilización de la lengua escrita en la realización de tareas de aprendizaje» citándose expresamente habilidades de realización de esquemas, guiones, resúmenes o subrayado. Es decir, se resalta el aspecto de habilidad o destreza que tiene la comprensión lectora, lo cual es el reconocimiento de la funcionalidad de la lectura como medio de aprendizaje en una cultura letrada como la nuestra. Ahora bien, el problema surge cuando se constata que la comprensión lectora es un proceso interactivo en el que intervienen tres elementos indisociables: el texto, el lector y el propósito de la lectura. Es decir, no se puede hablar de una comprensión lectora en abstracto sino contextualizada en un sujeto con unos conocimientos y unas estrategias determinadas que se enfrenta a unos determinados textos con finalidades muy diversas. En el caso que nos ocupa nos encontramos con un lector que será más o menos hábil con textos de carácter literario o con tareas de comprensión relativamente simples, como recordar determinados nombres o fechas, pero que puede ser poco diestro para estudiar o aprender a partir de textos informativos de ciencias sociales o naturales, que es donde habitualmente surge el problema. Es decir, lo que se hace didácticamente es enmarcar la comprensión lectora en el área de lengua y literatura, se practica esta comprensión fundamental, aunque no exclusivamente, con textos de carácter literario, y luego se pretende que el niño transfiera esas habilidades a otros contextos considerablemente distintos. En suma, a nuestro modo de ver, la situación es problemática ya que la comprensión lectora se descontextualiza en una de sus finalidades primordiales, el aprendizaje a partir de textos; no se enseñan estrategias y destrezas específicas, y por último, dejamos al alumno al azar de un autoaprendizaje por medio de ensayo y error que, en el mejor de los casos, le hace desperdiciar energías inútilmente, y en el peor le conduce a fracasos en su aprendizaje.

\section{OBJETIVOS Y BASES PSICOPEDAGOGICAS DEL PROGRAMA}

El programa que aquí presentamos pretende aportar una solución a la necesidad de enseñanza de destrezas de comprensión lectora en textos informativos típicos de las áreas de ciencias naturales y sociales. Dicho programa ha sido aplicado con resultados positivos por uno de los autores del presente trabajo en un aula de $5 .^{\circ}$ de EGB (Vidal-Abarca, 1990) y posteriormente por un grupo de profesores de $4 .^{\circ}$ a $60^{\circ}$ de EGB con resultados igualmente satisfactorios. Con este programa se pretendía enseñar a los chicos tres destrezas generales: $1 .^{2}$ ) distinguir la información importante de aquella otra de menor importancia, $2 .^{2}$ ) adoptar estrategias para organizar y estructurar adecuadamente la información que los textos proporcionan y 3. `) conseguir destrezas metacognitivas de control y regulación de la propia 
comprensión. Es decir, se partía de la base de que la comprensión es un proceso interactivo (Alonso, Mateos, 1985) en el cual el lector ha de construir una representación organizada y coherente del contenido del texto relacionando la información del pasaje con los esquemas de conocimiento previo de los niños, bien sea los esquemas relativos al conocimiento específico del contenido del texto (esquema de «ser vivo", de "suelo", de "medios de transporte», etc.) o bien aquellos otros esquemas acerca de la organización general de los textos informativos (textos que "comparan» objetos, textos que "clasifican o enumeran" cosas, etc.). En la medida en que los chicos son conscientes de estos esquemas de conocimiento, pueden adoptar estrategias para organizar y estructurar la información con el fin de obtener una representación coherente, ordenada y jerárquica, lo cual posibilita el aprendizaje a partir del texto. Asimismo, se partía de la base de que la comprensión es un proceso activo en el cual los sujetos pueden planificar, dirigir, comprobar, revisar y evaluar todos y cada uno de los pasos que les conducen a una comprensión efectiva, es decir, pueden controlar y regular su propia comprensión mediante esas destrezas metacognitivas (Baker y Brown, 1984).

Unos objetivos como los que acabamos de señalar requieren una metodología de instrucción basada en unos principios que son una síntesis de procedimientos de instrucción directa (Baumann, 1985), enseñanza explícita (Pearson y Dole, 1987) y enseñanza recíproca (Palincsar y Brown, 1984) los cuales resumimos a continuación:

1. Proporcionar objetivos claros a los alumnos. Dado que los niños han de realizar un aprendizaje activo de regulación y control de la propia comprensión, el profesor ha de comunicar de forma clara los propósitos y la funcionalidad de los aprendizajes.

2. El profesor ba de modelar las operaciones que realizarán los alumnos. Si en cualquier método de enseñanza el profesor ha de proporcionar un modelo claro de las destrezas que se instruyen en este caso, dado que se trata de operaciones mentales internas, es de la máxima importancia que se realice un modelado explícito sobre las operaciones mentales que los alumnos han de realizar.

3. La instrucción se ba de realizar en contextos reales en los que se favorezca la generalización a situaciones ordinarias de enseñanza y aprendi$z a j e$. Es decir, los textos y las situaciones de enseñanza serán lo más parecidos posible al contexto real en que se realiza la enseñanza.

4. El profesor debe proporcionar retroalimentación sobre los procesos de comprensión. Así, no se trata tanto de decir a los niños si sus respuestas son correctas o no cuanto de que sean conscientes de la adecuación de los. procesos que están poniendo en marcha en la tarea requerida y de las razones de sus respuestas con el fin de que las evalúen, corrigiendo aquellas que sean inadecuadas.

5. Se debe proporcionar una retroalimentación que favorezca la motivación y las atribuciones de los alumnos hacia el logro. Es decir, se trata de que los niños tomen el control de su propio aprendizaje en lugar de que dicho control resida en el profesor. Esto es particularmente importante en aquellos alumnos que tienen dificultades de aprendizaje, para los cuales es esencial que atribuyan sus propios progresos a su esfuerzo y a su mejora 


\section{6}

en estrategias de comprensión, lo cual se traduce en un incremento de la motivación.

6. Se debe promover el diálogo y la discusión tanto profesor-alumno como alumno-alumno. Es decir, se trata de promover la actividad en el aprendizaje, de forma que los niños, mediante la interacción y el diálogo acerca de sus respuestas explícitas y de sus procesos de pensamiento, vayan construyendo sus propios esquemas de comprensión de textos.

\section{DESCRIPCION DEL PROGRAMA}

El programa tal como se puede ver en la Figura 1 está estructurado en doce sesiones de una duración aproximada de 45 minutos que es el tiempo efectivo ordinario de una clase a razón de dos sesiones por semana. Los pasajes que los niños leían fueron adaptados de los libros de texto del área de experiencias social-natural de diversas editoriales con el fin de mejorar su comprensibilidad. Todos ellos tenían una estructura comparativa o enumerativa, es decir, eran textos que comparaban las características de dos clases de objetos (animales y vegetales, la España húmeda y la España seca, etc.), o bien eran textos que enumeraban y explicaban distintas clases de objetos pertenecientes a un mismo tópico (tipos de suelo, medios de transporte, etc.). Escogimos estas estructuras textuales debido a que eran las más frecuentes en los libros de texto de estos niveles y además porque diversas investigaciones han encontrado que son las más asequibles a los ninos de estas edades aunque sobre este punto no hay unanimidad (Richgels y col., 1987). Así, obviamos otras estructuras como las de tipo causativo o las de tipo descriptivo que son más frecuentes en niveles superiores de enseñanza. Los textos tenían una longitud que oscilaba entre 100 y 150 palabras que es el tamaño usual de los pasajes en que se suele dividir una lección en los libros de texto de $4 .^{\circ}$ y $5 .^{\circ}$ de EGB.

En cada una de las sesiones se realizaban diversas actividades con las que se pretendía que los alumnos pusieran en marcha determinadas operaciones cognitivas con la guía del profesor. Así, en las dos primeras sesiones pretendiamos que los niños identificaran la idea principal de un texto y los detalles que la apoyaban. Para ello, tras explicar los objetivos que perseguíamos así como su utilidad en la comprensión y el aprendizaje, dividíamos el texto en partes pidiéndoles que las leyeran y a continuación escribieran «qué decía» el pasaje en cada una de ellas (ver Figura 2). A continuación proponíamos encontrar un título, para lo cual pediamos que pensaran «de qué trataba» el pasaje en su conjunto y lo plasmaran en un título adecuado para la lectura. Posteriormente los niños releían lo que habían escrito anteriormente con el fin de identificar qué frase del texto expresaba lo más esencial que «decía» el pasaje en su conjunto. Dicha frase debería ser una oración no muy larga y tendría que expresar el contenido de la totalidad del texto. Como se puede apreciar, distinguíamos entre el «tema" o título que nos indica de qué trata el texto, y la «idea principal» o frase que indica qué dice el pasaje sobre ese tema. A continuación se pedía a los niños que escribieran o seleccionaran algún detalle o información menos importante que fuera coherente con esa idea principal y nos concretara algún aspecto de la misma. En todo este proceso se promovía el diálogo de forma 
FIGURA 1

\begin{tabular}{|c|c|c|c|}
\hline SESION & $\begin{array}{l}\text { ESTRUCTURA } \\
\text { TEXTUAL }\end{array}$ & ACTIVIDADES & $\begin{array}{c}\text { FASE } \\
\text { INSTRUCCIONAL }\end{array}$ \\
\hline $2^{1^{a}}$ & & $\begin{array}{l}\text { 1. Identificar la idea principal explicita. } \\
\text { 2. Identificar detalles de apoyo. } \\
\text { 3. Representar la macroestructura tex- } \\
\text { tual. } \\
\text { Las mismas }\end{array}$ & $\begin{array}{c}\text { Modelado } \\
\text { Práctica guiada }\end{array}$ \\
\hline $\begin{array}{l}3^{a} \\
4^{a}\end{array}$ & $\begin{array}{l}\text { COM- } \\
\text { PARA- } \\
\text { TIVA }\end{array}$ & $\begin{array}{l}\text { 1. Producir la idea principal explicita. } \\
\text { 2. Identificar detalles de apoyo. } \\
\text { 3. Representar la macroestructura tex- } \\
\text { tual. } \\
\longrightarrow \text { Las mismas }\end{array}$ & $\begin{array}{c}\text { Modelado } \\
\text { Práctica guiada }\end{array}$ \\
\hline $5^{\mathrm{a}}$ & & $\begin{array}{l}\text { Producir un texto con estructura textual } \\
\text { comparativa. }\end{array}$ & $\begin{array}{l}\text { Modelado y } \\
\text { práctica guiada }\end{array}$ \\
\hline $6^{a}$ & & $\begin{array}{l}\text { Combinación de actividades de las se- } \\
\text { siones } 1^{\mathrm{a}} \text { a } 4^{\mathrm{a}}\end{array}$ & $\begin{array}{l}\text { Práctica inde- } \\
\text { pendiente }\end{array}$ \\
\hline $7^{a}$ a $9^{a}$ & $\begin{array}{l}\text { ENU- } \\
\text { MERA- } \\
\text { TIVA }\end{array}$ & $\begin{array}{l}\text { Las mismas que en las sesiones } 1^{a} \text { a } 5^{a} \\
\text { y además: } \\
\text { 1. Ordenar oraciones textuales según } \\
\text { su importancia en el pasaje. } \\
\text { 2. Detectar oraciones consistentes e } \\
\text { inconsistentes con la idea principal. }\end{array}$ & $\begin{array}{c}\text { Modelado } \\
y \\
\text { práctica guiada }\end{array}$ \\
\hline $10^{\mathrm{a}} \mathrm{a} 12^{\mathrm{a}}$ & $\begin{array}{l}\text { ENUME- } \\
\text { RATIVA } \\
\text { Y } \\
\text { COMPA- } \\
\text { RATIVA }\end{array}$ & $\begin{array}{l}\text { Combinación de las actividades de las } \\
\text { sesiones anteriores y además: } \\
\text { 1. Distinguir entre textos con estructura } \\
\text { texlual comparativa y enumerativa. } \\
\text { 2. Representación mental de la macro- } \\
\text { estructura del pasaje. } \\
\text { 3. Resumen del contenido del pasaje. }\end{array}$ & $\begin{array}{l}\text { Modelado, } \\
\text { práctica guiada } \\
\text { y práctica } \\
\text { independiente }\end{array}$ \\
\hline
\end{tabular}

Esquema general del programa

que interviniera el mayor número posible de niños, se preguntaban las razones de las propias respuestas, se pedía opinión a los chicos sobre las respuestas y razones que habían dado otros compañeros, advirtiéndoles que no se trataba de sancionar sino de pensar conjuntamente, e insistíamos en que las respuestas adecuadas podían tener diversas formulaciones, es decir, que lo correcto no eran las frases sino las ideas que se expresaban por medio de ellas. En este sentido advertimos al lector que las respuestas que figuran en letra cursiva no son obviamente la única redacción correcta sino una de las posibles. Tras las dos actividades que acabamos de explicar se pasaba a la tercera, en la cual se pedía a los chicos que representaran la macroestructura textual, es decir, que produjeran una representación organizada y jerárquica de las ideas del pasaje. Para esta representación utilizamos la imagen de una "balanza" sirviéndonos de la idea analógica de "comparación" que suscita este instrumento. Así, los chicos completaban el diagrama con la información del texto y el profesor les hacía caer en la cuenta de cómo la idea principal de la lectura coincidía con los letreros colocados en la «balanza»: comparación-animales-vegetales, lo que equivale a decir 
FIGURA 2

LECTURA. tales.

Los seres vivos se clasifican en animales y vege-

Los animales necesitan moverse para buscar el alimento, por lo que tienen los sentidos, como el olfato - la vista, muy desarrollados. Sin embargo los vegetales no tienen movilidad, pues no necesitan desplazarse para buscar el alimento, ni tienen los órganos de los sentidos tan desarrollados como los animales.

Otra diferencia importante entre animales y vegetales se refiere a la función de la nutrición. Los animales necesitan alimentos orgánicos como carne, pescado, frutas o verduras, mientras que los vegetales absorben alimentos minerales como agua a sales miinerales por medio de las raices y las hojas.

Así, podemos decir que los animales y los vegetales se diferencian por las funciones de relación y nutrición.
1) Escribe de forma breve qué se dice en cada una de las partes de la lectura.

Parte 1. Los seres vivos se clasifican en animales y vegetales. Los animales so mueven

.................................... y tienen los sentidos desarrollados mientras que tos vegetales no se mueven ni tienen desarrollados los sentidos

Parte $2^{2}$ Los animales se alimentan de torma diferente a los vegetales Los animales y ve... getales se diferencian por las funciones de ....relación y nutrición.

2) ¿Qué titulo podriamos poner a la lectura? Escribelo con letras mayúsculas. LOS ANIMALES YLOS VEGETALES

3) La Idea Principal de la lectura es: Los animales y los vegetales se diferencian por las funciones de relación y nutrición.

4) Por qué te parece esa la Idea Principal de la lectura? Porque esa frase recoge el contenido de toda la lectura, de la primera y la segunda parte.

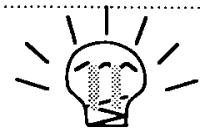

5) Subraya alguna frase de la lectura que te parezca interesante con respecto a la diferencia entre los a-

nimales y vegetales. (Los niños subrayarán alguna diferencia realativa a las funciones de relación o de nutrición. Se tendrá en cuenta que se subrayen caracteristicas tanto de animlaes como de vegetales).

6) Completa el siguiente gráfico para representar cómo están organizadas las ideas de la lectura.

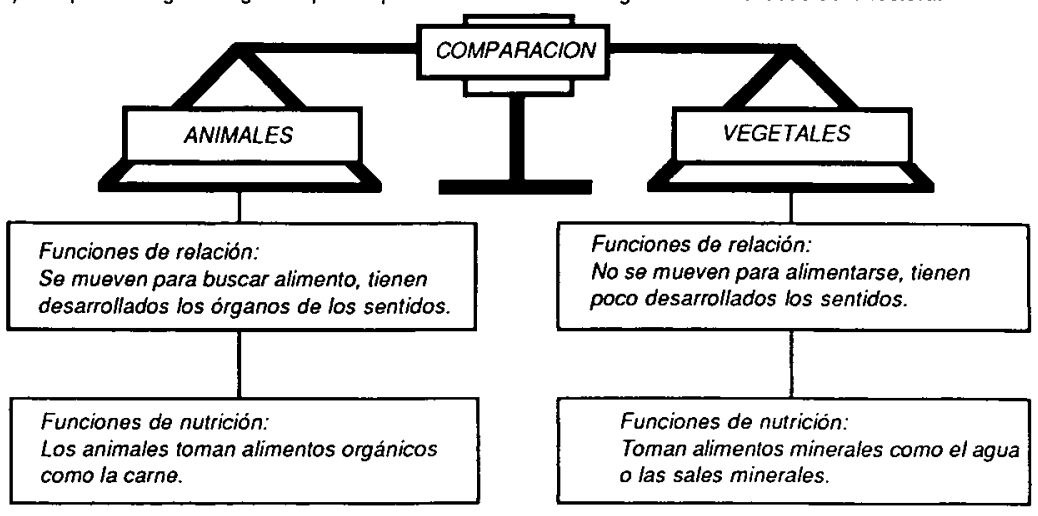

Ejemplo de actividades realizadas en la primera parte del programa

que el texto está explicando las diferencias (y quizá las semejanzas) entre estos dos tipos de seres vivos.

Estas actividades que acabamos de describir eran modeladas por el profesor en la primera sesión, planteándose él mismo preguntas, verbalizando las respuestas ante los niños, y promoviendo que estos se plantearan las preguntas y contrastaran sus soluciones con las que el profesor iba verbalizando. En la segunda sesión el maestro guiaba los procesos de pensamiento, pero eran los niños los que debian ir dando las respuestas y dialogando acerca de ellas. La tercera y la cuarta sesión eran muy similares a las dos primeras con la salvedad de que los textos no presentaban ninguna frase de la 
que se pudiera decir que era la idea principal sino que los niños debían «inventarla", lo cual se advertía previamente a los muchachos. La quinta sesión estaba dedicada a examinar cómo se había construido un texto comparativo con el fin de que los alumnos posteriormente elaboraran otro diferente sobre un tópico que ellos elegían. Para ello el profesor explicaba cómo cuando un autor quiere hacer un texto comparativo ha de pensar qué es lo que quiere comparar, y qué características son las que diferencian o asemejan a esos objetos. A continuación se presentaba un ejemplo concreto, uno de los textos ya leídos en sesiones anteriores, mostrando la imagen de la «balanza» rellena con los datos que deseábamos incluir y modelando cómo a partir de ahí se elaboraba el texto correspondiente. Tras aclarar las dudas, proporcionábamos una hoja con las reglas que había que seguir y se pedía a los niños que individualmente o por parejas elaboraran un texto de comparación con la idea principal explícita, pero teniendo en cuenta dónde la iban a colocar (al comienzo, al medio o al final), ya que el lugar donde la colocaran influiría para facilitar o dificultar la comprensión y el recuerdo del texto. Finalmente, en la sexta sesión los niños tenían que encontrar o producir la idea principal, identificar detalles y representar la macroestructura textual de dos textos, uno con la idea principal explícita y otro con idea principal implícita. En ambos casos la intervención del profesor era escasa, prestando únicamente ayuda a los niños de forma individual, ya que se trataba sobre todo de revisar la eficacia de la enseñanza impartida con el fin de tomar medidas correctoras y atender a dificultades individuales.

Las sesiones séptima, octava y novena eran muy similares a las cinco primeras pero empleando pasajes con estructura textual enumerativa y con unos cambios que a continuación pasamos a detallar. En la sesión séptima se presentaban dos textos, en lugar de uno como en las anteriores. En cada uno de ellos las actividades eran idénticas sirviendo el primero para el modelado del profesor y el segundo para la práctica guiada. El primer cambio introducido en estas sesiones consistió en que los niños leían el texto de una vez sin dividirlo en partes, tal y como se puede ver en la Figura 3. Tras dicha lectura se pensaba y escribía un título adecuado para el pasaje. A continuación se buscaba la frase textual que pudiera expresar la idea principal de la lectura. Posteriormente se introducían dos nuevas actividades, la primera de las cuales consistía en puntuar con 2, 1 ó 0 tres frases de la lectura según expresaran ideas más o menos importantes con el fin de ampliar la habilidad de jerarquización de ideas del texto, favoreciendo así la formación de la macroestructura textual. La segunda actividad incluida consistió en seleccionar detalles que fueran congruentes con una determinada idea principal. En este caso (ver Figura 3) dicha idea principal tenía estructura enumerativa con lo cual aquellas ideas que no se correspondieran con esta estructura no deberían ser seleccionadas como congruentes. De esta forma se favorecía la formación de esquemas de conocimiento sobre las estructuras textuales de la prosa. La última actividad consistía en representar la macroestructura textual sirviéndonos en este caso de la figura de un «árbol» en cuyo tronco se representaba la idea principal, representando en las ramas principales las diferentes clases enumeradas y en las ramas secundarias los detalles (ver Figura 3).

La sesión octava era idéntica a la séptima con la diferencia de que los 
FIGURA 3

LECTURA

Cuando sales por el campo habrás podido obsenvar que hay zonas en las que abunda un tipo de plantas y árboles mientras que en otras la vegetación es distinta $o$, en ocasiones, crecen únicamente algunos matorrales. Eso es debido, entre otras causas, a los tipos de suelo que hay en nuestro pais.

Un tipo de suelo es el arcilloso. Se llaman así porque en ellos predomina la arcilla. Estos suelos necesitan abundante agua para que en ellos crezcan las plantas.

Otra clase son los suelos arenosos en los que predomina la arena. Generalmente son pobres en vegetación. El pino pin̄onero crece en suelos arenosos ya que necesita poca agua para desarrollarse.

Un tercer tipo de suelo es el llamado "suelo salino". En los suelos salinos abunda mucho una clase de sales, como es por ejemplo la sal común. El hecho de que abunden las sales hace que estos suelos no sean buenos para la vegetación.

Asi, pues, podemos distinguir varios tipos de suelo según los materiales que predominan en su composicion.

1) Si tuviéramos que poner un titulo breve a la lectura, ¿cuál le pondriamos? Escribelo con mayúsculas. LOS TIPOS DE SUELO

2) ¿Cuál es la oración de la lectura que nos indica la Idea Principal? Podemos distinguir varios tipos de suelos según los materiales que predominan en su composición.

3) Como ya sabes, en las lecturas hay oraciones más y menos importantes. A continuación tienes tres oraciones. Coloca un 2 junto a la más importante, un 1 junto a la que sea un poco menos importante y un 0 junto a la que sea solamente un detalle.

....L Una clase de suelo es el arenoso en el que predomina la arena.

....2 Podemos distinguir varios tipos de suelos según los materiales que predominan en su composición.

.... El pino piñonero crece en suelos arenosos ya que necesita poca agua para desarrollarse.

4) Lee la oración que está en negrita. Marca con una cruz cuál de las otras oraciones podria ir con ella. Podemos distinguir varios tipos de suelos según los materiales que predominan en su composición.

Un tipo de suelo es el arenoso, donde crecen pinos

$\square$ As tierras de huerta tienen suelos muy buenos

$X$ e llama suelo arcilloso aquél en el que predomina la arcilla

$\square$ Con la arcilla se pueden hacer figuras de adorno muy bonitas

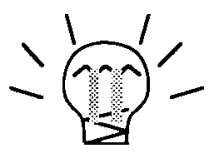

5) Completa el siguiente gráfico para representar cómo están organizadas las ideas de la lectura.

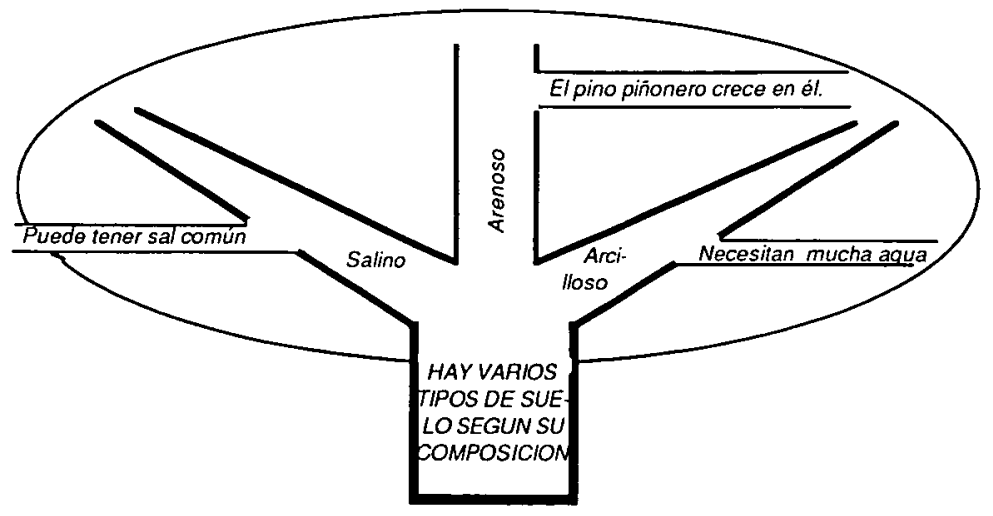

Ejemplo de las actividades realizadas en la segunda parte del programa

textos tenían la idea principal implícita y los niños tenian que «inventarse» aquella que el autor podia haber escrito para facilitar la comprensión y el aprendizaje. La sesión novena era paralela a la quinta, es decir, se enseñaba a los niños cómo producir un texto de tipo enumerativo, se le proporcionaban unas reglas para hacerlo, pidiéndoles posteriormente que lo elaboraran. En las tres últimas sesiones se realizaban actividades similares a las des- 
critas anteriormente junto a otras nuevas, empleando dos textos en cada sesión, uno de tipo enumerativo y otro comparativo. En la sesión décima los niños leían los dos textos y a continuación identificaban la estructura textual de cada uno de ellos tal como se puede ver en la primera parte de la Figura 4. A continuación los chicos realizaban las actividades comentadas anteriormente con cada uno de los pasajes. En las sesiones undécima y duodécima se introdujeron dos nuevas actividades. La primera de ellas consistía en formar una representación mental de la macroestructura textual, para lo cual se pedía a los niños que se imaginaran la "balanza» o el "árbol" rellenos con los datos de la lectura (ver Figura 4). Para ayudar a esta representación se les preguntaba cuántas ramas tenía el «árbol» (o bien cuántos cajones tenía la «balanza») y se establecía un diálogo acerca del contenido de las mismas. De esta forma se interiorizaba la macroestructura textual y se preparaba a los niños para que realizaran las operaciones mentales correspondientes cuando se enfrentaran a los textos habituales de estudio. La segunda actividad consistía en elaborar un resumen del contenido del pasaje. Se advertía que dicho resumen debía ser realizado a partir de la representación mental anterior. Primeramente se dialogaba acerca de las ideas que debía contener y, posteriormente, tras llegar a un acuerdo global, cada uno de los chicos elaboraba el suyo constrastando los resultados y los procesos mentales correspondientes.

\section{CONTEXTUALIZACION DE LAS DESTREZAS DE COMPRENSION}

El programa descrito únicamente pretende poner en marcha unos procesos de comprensión, es decir, provocar una forma nueva de acercamiento a los textos informativos concretado en unas operaciones mentales que los chicos deben aplicar en las situaciones ordinarias de aprendizaje. Quizá sea patente para los lectores que el programa igualmente supone un acercamiento distinto a la enseñanza de la comprensión y, en general, a la enseñanza de aprendizajes a partir de textos escritos. En este sentido, las operaciones mentales aprendidas y los procedimientos de enseñanza practicados deben ser generalizados y aplicados en las situaciones ordinarias de clase. Es por ello que debería quedar claro que no se trata de un programa para aplicar en el área del lenguaje, sino más bien en las áreas de contenido informativo como son las ciencias sociales y naturales. Ahora bien, esa generalización tropieza al menos con dos dificultades de las que no tenemos experiencia directa ya que, de momento, no hemos realizado ningún estudio sistemático al respecto.

La primera dificultad se refiere a que los textos de las distintas editoriales generalmente no suelen ser tan «comprensibles» como los empleados en el programa de entrenamiento ni tampoco suelen responder a una estructura textual tan definida. A pesar de todo ello creemos que los procesos mentales aprendidos son lo suficientemente flexibles como para acomodarse a textos naturales siempre que el profesor guie y promueva dicha acomodación. La segunda dificultad se refiere a las actividades de comprensión y aprendizaje que presentan los libros de texto. En general estas actividades se dirigen a cuestiones de detalle obviando en gran medida activi- 
FIGURA 4

LECTURA1.

Uno de los hechos más característicos de nuestra época son los viajes. Hoy en dia se viaja mu cho más que en cualquier otra época de la historia. Ello es debido, sobre todo, a los modernos me-dios de transporte.

Hay un tipo de transporte que va por la tierra. Son los medios de transponte terrestre. Los más modernos son bastante rápidos. Entre ellos podemos citar al coche, el autobús o el camión. Se utilizan para el traslado de viajeros y mercancias.

Los medios de transporte maritimo, como el barco, son muy antiguos, aunque en la actualidad han aumentado considerablemente su tamaño y su potencia. Se utilizan, sobre todo. para el transporte comercial entre diferentes paises o entre islas.

Un tercer tipo de transporte es el aéreo. Es el medio de transporte más reciente. Su gran ven- taja sobre los demás es la rapidez. El avión anglo-frances "Concorde" va de Paris a Nueva York en unas pocas horas.

LECTURA 2

Seguramente habrás visto volar cerca de la luz por las noches a unos animalitos que se parecen a las mariposas. Son las polillas de la luz, y no se deben confundir con las mariposas.

Una primera señal que te puede servir para diferenciar estos tipos de animalitos es el momento del dia en que las veas volar. Mientras que las mariposas suelen volar por el dia, las polillas de la luz lo suelen hacer por las noches.

Otra diferencia muy llamativa es el color que tienen sus alas. Las mariposas suelen tener unos co lores muy bonitos y vivos. Hay mariposas rojas, amarillas, verdes, blancas o de varios colores mezclados. Sin embargo las polillas de la luz tienen colores más oscuros.

El cuerpo también es diferente en ambos animales. El cuerpo de las mariposas es más delgado y no está cubierto de pelillos. Por otra parte. las antenas que tiene en la cabeza la polilla de la luz son más gruesas y no tienen un pequeño bulto en los extremos, como ocurre con las mariposas.

1) ¿Cuál de las lecturas COMPARA $\infty$ sas? ¿Y cuál ENUMERA cosas? Une con flechas la respuesta correcta:

LECTURA 1

LECTURA 2
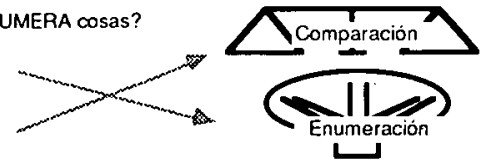

Piensa en la LECTURA 1. Imaginate el ARBOL RELLENO con los datos de la lectura y CONTESTA.

2) ¿Cuántas ramas tiene el ARBOL que te has imaginado? .......Tres ¿Por qué? Porquela lectura nos habla de tres medios de tres medios de transporte

3) ¿Está escrita la ldea Principal en la Lectura? ....... No.

4) Inventate la oración que deberia haber escrito el autor de la lectura para indicarnos la Idea Principal.

Hoy dia existen tres medios de transpoorte modernos: terrestres marimimos y aéreos

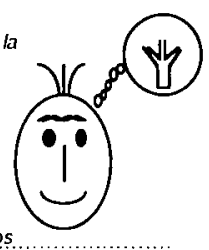

5) Lee las oraciones que están escritas a continuación. Coloca un 1 junto a las que sean bastante importantantes y un 0 junto a las que sean detalles.

I. Hoy dla se viaja mucho debido a los modernos medios de transporte.

l... Hay un medio de transporte que va por tierra.

l.. Otro medio de transporte es el maritimo.

..o. Antiguamente también habia barcos más lentos y menos potentes.

6) Piensa en la idea principal y en cómo está organizada la lectura. A continuación escribe un RESUMEN de la lectura donde expreses no sólo la idea principal sino otras informaciones que sean importantes. Aclualemente hay unos medios de transporte que son de tres tipos: el terrestre que es bastante rapido y se utiliza para viajeros y mercancias, el maritimo, que se emplea sobre todo para el conercio y el transporte aéreo, que es mucho más ràpido que los demás.

Ejemplo de las actividades realizadas en la tercera parte del programa

dades que impliquen la formación de la macroestructura textual (Solé, 1987), o bien, cuando las incluyen (por ejemplo pidiendo a los niños que escriban resúmenes o esquemas) no suelen proporcionar indicaciones acerca de cómo realizarlas. Es decir, si el profesor se atiene a las actividades que proponen los libros de texto difícilmente podrá generalizar las destrezas adquiridas en el programa y, por tanto, el entrenamiento perderá efi- 
cacia y aparecerá como algo añadido y no integrado en el currículum ordinario, que es lo que se pretende conseguir.

¿Cómo se puede promover esa generalización que estamos propugnando? En primer lugar creemos importante que los principios metodológicos que explicamos anteriormente estén presentes en la intervención docente en las clases ordinarias. En segundo lugar se debe ser consciente de que el resultado de la comprensión y del aprendizaje depende de las operaciones mentales que realiza el sujeto cuando se enfrenta a un texto. Así, si se pretende que los chicos formen la macroestructura del texto, es decir, obtengan una representación mental coherente, ordenada y jerárquica de las ideas que el autor quiere transmitir, ello se favorecerá promoviendo actividades tales como las descritas en el programa (producir títulos, escribir la idea principal, graduar la importancia de las oraciones del texto, elaborar resúmenes, etc.) y otras como predecir el contenido del texto a partir de títulos o de los párrafos iniciales, responder a preguntas de inferencia, o elaborar esquemas. En tercer lugar, dado que en la generalización se deben emplear textos ordinarios, habría que enseñar a los niños aquellas otras estructuras textuales que estuvieran presentes en dichos textos (p.e. secuencia, causación, descripción, etc.; ver Sánchez, 1989, Experimentos II y III), así como también se podría incluir enseñanza específica sobre algunas dificultades de comprensibilidad de los textos, siempre de forma adaptada a las posibilidades cognitivas de los niños.

\section{Referencias}

Alonso, J. y MATEOS, M. M. (1985). Comprensión lectora: modelos, entrenamiento y evaluación. Infancia y Aprendizaje, 31-32, 5-19.

BAKER, L y BROWN, A. L. (1984). Metacognitive skills and reading. En P.D. Pearson (ed.), Handbook of reading research. Nueva York, Longman.

BAUMANN, J. F. (1985). La eficacia de un modelo de instrucción directa en la enseñanza de la comprensión de ideas principales. Infancia y Aprendizaje, 31-32, 89-105.

MEC (1989). Diseño Curricular Base: Educación Primaria. Madrid. Servicio de Publicaciones del MEC.

MEC (1989). Diseño Curricular Base: Educación Secundaria Obligatoria. Madrid. Servicio de Publicaciones del MEC.

PALINCSAR, A. S. y BROWN, A. L. (1984). Reciprocal teaching of comprenhension-fostering and comprehension-monitoring activities. Cognition and Instruction, 1 (2), 117-175.

PeARSON, P. D. y DOLE, J. A. (1987). Explicit comprehension instruction: a review of research and new conceptualizations of instruction. The Elementary School Journal, 88, 2, 151-175.

RichGels, D. J.; McGeE, L. M.; Lomax, R. G. y ShEARD, C. (1987). Awareness of four text structures: effects on recall of expository text. Reading Research Quarterly, XXII, 2, 177-196.

SÁNCHEZ, E. (1989). Procedimientos para instruir en la comprensión de textos. Madrid, Ministerio de Educación y Ciencia, CIDE.

SOLE, I. (1987). L'ensenyament de la comprensió lectora. Barcelona, Ceac.

VIDAL-ABARCA, E. (1990). Un programa para la enseñanza de la comprensión de ideas principales de textos expositivos. Infancia y Aprendizaje (en prensa). 


\section{4}

\section{Comprender para aprender: Un programa para mejorar la comprensión y el aprendizaje de}

textos informativos. E. Vidal-Abarca y R. Gilabert.

CLEE, 1990, 6, pp. 113-124

\section{Resumen}

Uno de los objetivos más importantes de la escolaridad obligatoria es conseguir que los alumnos sean capaces de aprender a partir de textos escritos. Sin embargo, son muchos los chicos que no llegan a conseguir este objetivo, al menos de una forma satisfactoria a juicio de los profesores. Este trabajo analiza algunas de las causas posibles de este problema y describe un programa de intervención para enseñar a los muchachos a comprender $y$ aprender a partir de textos informativos. En este programa son esenciales tanto La metodología de instrucción que se adopta como las actividades que el profesor preenta a los niños.

Datos sobre los autores: Ambos autores, primero como maestros y posteriormente como profesores en la EU de Formación del profesorado de EGB, están trabajando desde hace varios años en temas de lectura y escritura, habiendo centrado sus intereses en los últimos años en la comprensión lectora orientada al aprendizaje de textos.

Dirección: Universidad de Valencia. EU de Formación de Profesorado de EGB. Alcalde Reig 8, 46006 Valencia.

(C) de todos los artículos. Deberá solicitarse por escrito autorización de CL\&E y de los autores para el uso en forma de facsímil, fotocopia o cualquier otro medio de reproducción impresa. CL\&E se reserva el derecho de interponer acciones legales necesarias en aquellos casos en que se contravenga la ley de derechos de autor. 